\title{
The Diurnal Project: Diurnal and Circadian Expression Profiling, Model-based Pattern Matching, and Promoter Analysis
}

\author{
T.C. Mockler, ${ }^{*}$ T.P. Michael ${ }^{\dagger}$ H.D. Priest, ${ }^{*}$ R. Shen, ${ }^{*}$ C.M. Sullivan, ${ }^{*}$ \\ S.A. GIVAN, ${ }^{*}$ C. MCENTEE, ${ }^{\dagger}$ S.A. KAY ${ }^{\ddagger}$ AND J. CHORY ${ }^{\dagger}$ \\ *Department of Botany and Plant Pathology and Center for Genome Research and Biocomputing, \\ Oregon State University, Corvallis, Oregon 97331; ${ }^{\dagger}$ Plant Biology Laboratory, The Salk Institute for \\ Biological Studies, La Jolla, California 92037; Department of Cell and Developmental Biology, \\ University of California at San Diego, La Jolla, California 92093-0116; ${ }^{\S}$ Howard Hughes Medical \\ Institute, The Salk Institute for Biological Studies, La Jolla, California 92037
}

\begin{abstract}
The DIURNAL project (http://diurnal.cgrb.oregonstate.edu/) provides a graphical interface for mining and viewing diurnal and circadian microarray data for Arabidopsis thaliana, poplar, and rice. The database is searchable and provides access to several user-friendly Web-based data-mining tools with easy-to-understand output. The associated tools include HAYSTACK (http://haystack.cgrb.oregonstate.edu/) and ELEMENT (http://element.cgrb.oregonstate.edu/). HAYSTACK is a modelbased pattern-matching algorithm for identifying genes that are coexpressed and potentially coregulated. HAYSTACK can be used to analyze virtually any large-scale microarray data set and provides an alternative method for clustering microarray data from any experimental system by grouping together genes whose expression patterns match the same or similar user-defined patterns. ELEMENT is a Web-based program for identifying potential cis-regulatory elements in the promoters of coregulated genes in Arabidopsis, poplar, and rice. Together, DIURNAL, HAYSTACK, and ELEMENT can be used to facilitate crossspecies comparisons among the plant species supported and to accelerate functional genomics efforts in the laboratory.
\end{abstract}

\section{INTRODUCTION}

Various databases containing gene expression data and Web-based tools for analyzing microarray data have emerged as valuable resources for many aspects of plant research. These resources include AtGenExpress (Schmid et al. 2005; http://www.weigelworld.org/resources/micro array/AtGenExpress/), ArrayExpress (Brazma et al. 2003; http://www.ebi.ac.uk/arrayexpress/), Botany Array Resource (BAR; Toufighi et al. 2005; http://www.bar. utoronto.ca/), GENEVESTIGATOR (Zimmermann et al. 2004; https://www.genevestigator.ethz.ch/), GEO (Barrett et al. 2007; http://www.ncbi.nlm.nih.gov/geo/), NASC Arrays (Craigon et al. 2004; http://affymetrix.arabidopsis. info/), PlexDB/Barleybase (Shen et al. 2005; http://www. plexdb.org/), TAIR (Garcia-Hernandez et al. 2002; Rhee et al. 2003; http://www.arabidopsis.org/), and VirtualPlant (http://virtualplant.org). Here, we introduce the DIURNAL project, consisting of a database containing diurnal and circadian expression data for approximately 22,800 Arabidopsis genes collected during 11 experiments, a Web interface for accessing this database, and complementary Web-based tools for analyzing microarray data and promoter sequences.

DIURNAL and its associated tools differ from other resources in several ways. DIURNAL is focused on plantdiurnal- and circadian-clock-regulated gene expression and currently supports Arabidopsis, rice, and poplar, three plant species with high-quality annotated genome sequences (Arabidopsis Genome Initiative 2000; Inter- national Rice Genome Sequencing Project 2005; Tuskan et al. 2006). A novel tool called HAYSTACK allows users to mine large microarray expression data sets by searching for specific user-defined patterns of expression. HAYSTACK is designed to find rare occurrences of very specific patterns in large data sets and provides an alternative method for clustering microarray data by grouping genes whose expression patterns match the same or similar HAYSTACK patterns. In addition to clustering genes based on the user-supplied model patterns, HAYSTACK can be used to identify genes that exhibit a pattern of expression similar to that of a particular gene of interest. Although we have used HAYSTACK in the DIURNAL project to identify diurnal and circadian regulated genes, this tool can be used to compare any large-scale data set representing at least three samples (e.g., treatments, genotypes, and time points) against a set of user-supplied model patterns. The resulting lists of coregulated genes in the HAYSTACK output can then be used with a third tool, ELEMENT, which is an enumerative promoter analysis program that analyzes the upstream regions of Arabidopsis, poplar, or rice genes to discover overrepresented elements that may represent novel transcriptionfactor-binding sites. Together, the DIURNAL suite of tools allows researchers to go from expression data to cisregulatory elements and place this into the context of diurnal and circadian control, an important layer of regulation in Arabidopsis, with $90 \%$ of genes exhibiting diurnal or circadian regulation under at least one growth condition (T. Michael et al., in prep.). 


\section{THE DIURNAL DATABASE}

Organisms experience daily environmental changes in light (photocycles) and temperature (thermocycles) that vary by season and latitude. Consequently, organisms have evolved an endogenous circadian clock with a period of about 24 hours, which ensures that internal biological processes are appropriately synchronized with the daily changes in the environment (Michael et al. 2003; Woelfle et al. 2004; Dodd et al. 2005). Transcript abundance can be found peaking at almost every hour during the day/night cycle, and this regulation forms the foundation for time-of day-specific biological activities.

The Arabidopsis microarray data available in the DIURNAL database was collected using the Affymetrix ATH1 GeneChip microarray platform, which represents about 22,800 genes as annotated by TAIR (ftp://ftp. arabidopsis.org/home/tair/Microarrays/Affymetrix). To identify cycling genes, we used these 336 model patterns with HAYSTACK to interrogate Affymetrix ATH1 GeneChip data sets for 11 time courses with 12 time points each. These data are presented and analyzed in a manuscript that has been submitted for publication $(\mathrm{T}$. Michael et al., in prep.). Plant material and growth conditions for the Arabidopsis time courses are described at http://diurnal.cgrb.oregonstate.edu/diurnal_details.html.

\section{Interface Design, Features, and Navigation}

Layouts, fonts, color schemes, and navigation were designed to make the interface user-friendly. The main page of DIURNAL contains a link to an "About Diurnal" page that summarizes the project. This page has a menu bar with links to the other tools of the project: ELEMENT and HAYSTACK. An additional tool, ORTHOMAP, provides predicted orthologs, homologs, or simple best BLAST matches for Arabidopsis, rice, and poplar and links to the respective external annotation resources at TAIR, TIGR, or JGI. This tool allows a user to obtain this information for a gene or group of genes of interest, without having to browse different databases or conduct sequence comparisons. The menu bar and links box appear on every page to enable efficient browsing between tools. In addition, the tools are connected in such a way that, for example, a list of genes identified by a query of DIURNAL or a list of predicted orthologs identified by a query of ORTHOMAP can be sent to ELEMENT for promoter analysis. The databases underlying DIURNAL, ELEMENT, and ORTHOMAP are based on MySQL, and the Web interfaces have been implemented using the Apache HTML delivery engine (http://www.apache.org) Perl and Mason (http://www.masonhq.com).

\section{DIURNAL: A Diurnal/Circadian Gene Expression Data-mining Tool}

DIURNAL is useful for a researcher who is interested in the diurnal or circadian expression profiles of a particular set of genes, either for diurnal/circadian experiments or for planning other types of experiments. The interface allows users to input a list of genes and select among the experiments in the database. The data resulting from the query is displayed graphically and available for download as a text file. In addition, we enable direct exporting of gene lists resulting from queries into ELEMENT for promoter analysis.

The entry page of DIURNAL includes a text box for entering locus identifiers and check boxes for selecting experimental conditions/array data sets. This page represents the "Basic Search," which has options for displaying the best-fitting HAYSTACK model and the expression profiles for multiple genes on a single graph, and for normalizing the expression levels among multiple genes being graphed. The normalization option makes it easier to compare the phases and waveforms of expression for sets of genes with large differences in expression levels. The basic search returns a graph or graphs of the diurnal or circadian expression profiles for the gene(s) submitted by the user (Fig. 1A). Below the graph is a table that summarizes the condition, phase, best-fitting model, and correlation value representing the quality of the match between the model and the experimental data. Above each graph are links to the raw text data and to the annotation page for the particular gene. By clicking on the graph, the user is presented with a larger version of the graph that can be downloaded in PNG image file format.

The left side of all pages within DIURNAL contains a menu box with a link to "Advanced Search." On the Advanced Search page, a user can query the database to return all genes matching a set of user-defined criteria. The user can define the phase, model, and correlation cutoff values and use a pulldown menu to select an experimental condition to query. Results are represented as a table listing genes matching the query criteria (Fig. 1B). Each row of the table represents a single gene and the fields of the table contain the Affymetrix probe-set identifier, the locus identifier, correlation value, phase of expression based on the best-fitting HAYSTACK model, and the name of the bestfitting model. The rows are linked to a detailed graphical display of the time course expression profile for the particular gene. As in the basic search, the graph image and raw data are downloadable, and a link is provided to the respective annotation database. An advanced search allows the user to cluster cycling probe sets/genes by their phase or waveform (best model) and thus can generate gene lists suitable for input to ELEMENT or another promoter analysis algorithm. To facilitate such analysis, we have provided an option to send the gene list resulting from a DIURNAL advanced search directly to ELEMENT for prediction of potential cis-regulatory elements.

When a user enters a list of genes and chooses the desired options, the microarray data are selected for display by looking up the appropriate Affymetrix probe-set identifier in a table within our database. There is not always a unique relationship between the Affymetrix probe-set identifier and a locus identifier, so more than one row of the table may correspond to the same gene, but representing data from different probe sets. The data generated by the query is displayed on the appropriate results pages, depending on the options chosen and whether a basic or advanced search was performed.

Another output option of DIURNAL is a tab-delimited 


\section{A Diurnal Results Page}

Welcome to the Diurnal Results page.

You can view the source data for any graph by clicking the header.

Both the graphs and the source data are downloadable, by right clicking on either the link or the graph, and selecing 'save as'. Also, you may click on any chart to enlarge it.

Gene: AT1G01060 [Graph source data - AT1G01060 Information at TAIR]

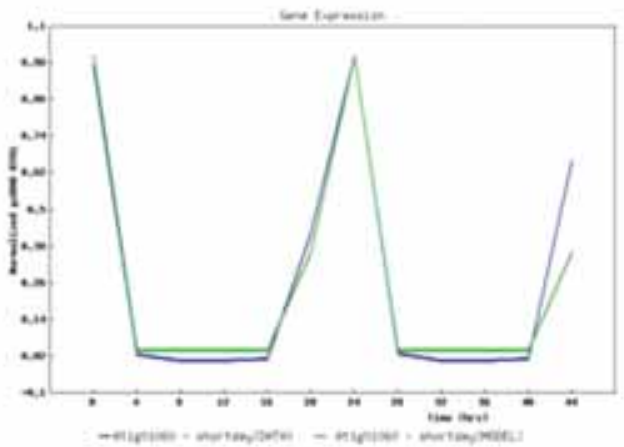

\begin{tabular}{|l|l|l|l|}
\hline Condition & Phase & Model & Correlation \\
\hline shortday & 23 & ct23-spike & 0.971688 \\
\hline
\end{tabular}

B

\begin{tabular}{|c|c|c|c|c|}
\hline Affy ID & Locus ID & Correlation & Phane & Best Model \\
\hline 267199_at & AT2030990 & 0.962899 & 13 & et13-spike \\
\hline 264482_at & AT1077210 & 0.962727 & 13 & et 13-spike \\
\hline 250777_at & Ar5005440 & 0.958629 & 13 & et 13-spike \\
\hline 256433_at & A23010985 & 0.951229 & 13 & et13-spike \\
\hline 262870_at & $\operatorname{Ar} 1064710$ & 0.945601 & 13 & et13-apike \\
\hline 266825_at & AT2022890 & 0.944285 & 13 & et13-spike \\
\hline 263461_at & AT2031800 & 0.943772 & 13 & et 13-spike \\
\hline 261668_at & AT1618500 & 0.942345 & 13 & et13-spike \\
\hline 252924_at & Ar4039070 & 0.940817 & 13 & et13-spike \\
\hline 258404_at & AT3017465 & 0.940213 & 13 & et13-apike \\
\hline 267523_at & Ar2G30600 & 0.939136 & 13 & et13-spike \\
\hline 259813_at & AT1649860 & 0.937439 & 13 & et13-spike \\
\hline 258531_at & Aร3606720 & 0.935411 & 13 & et13-spike \\
\hline 257206_at & AT3016530 & 0.934895 & 13 & et13-spike \\
\hline
\end{tabular}

Figure 1. DIURNAL. (A) Example of the output of the DIURNAL Basic Search showing a graph of a gene expression profile for AT1G01060 under the short-day condition with a summary of model match statistics. $(B)$ Example of the HTML table output from the DIURNAL Advanced Search showing genes and model match statistics fitting the search criteria. Clicking on a row of the table displays a graph for the gene and its best-fitting HAYSTACK model. 
plain text file that contains the expression profile data for queried probe sets/genes. The data presented in this file are the same data as those used to generate the graphs, with each row of data representing a single gene or probe set. The data columns list the probe-set identifier, locus identifier, best model, the experimental condition/data set, and correlation, whether the row is data (DATA) or fitted model (MODEL), followed by the series of expression values for the time course.

DIURNAL may be used in several different ways by a biologist. First, the tool can be used to characterize the diurnal or circadian temporal expression pattern of a gene of interest, especially by querying the diurnal conditions that approximate standard laboratory conditions such as short days or long days. Second, it can be used to identify genes coexpressed and potentially coregulated with a gene of interest. This type of analysis can suggest potential protein interactions as well because it has been demonstrated that gene expression among interacting proteins can coevolve (Fraser et al. 2004). In vivo experiments altering gene expression or using the two-hybrid system can further elucidate potential interactions among the genes identified. Finally, DIURNAL provides a method of clustering coexpressed genes whose promoters may be analyzed with either ELEMENT or other algorithms.

Because the DIURNAL interface can accept lists of multiple genes for comparison, it can also be used to predict functional redundancies among members of a gene family. For example, a user could submit a list of gene identifiers for a family of genes and use the DIURNAL output to classify them according to their temporal expression patterns. Example of such analyses are shown in Figure 2. Members of the C2C2-YABBY transcription factor family function to specify abaxial cell fate in Arabidopsis (Siegfried et al. 1999). A query of DIURNAL using the gene identifiers for the six members of the C2C2-YABBY TF family reveals diurnal expression profiles consistent with functional redundancy among some family members (Fig. 2A). Five out of six family members are represented on the Affymetrix ATH1 microarrays, and three out of these five cycle under the long-day condition. YAB1 (At2g45190) and YAB3 (At4g00180) share similar expression profiles, with peak expression occurring just before dawn, at phases 23 and 22, respectively. The similar phasing of expression of these two family members could indicate functional redundancy at this time of day. In contrast, YABBY5 (At2g26580) cycles with a phase of 16 hours after dawn, whereas CRC (AT1G69180) and YAB4 (AT1G23420) do not cycle. Another example involves an 11-gene family of trehalose-6-phosphate synthases involved in biosynthesis of trehalose, a sugar whose accumulation is implicated in drought stress tolerance (Leyman et al. 2001; Karim et al. 2007). All 11 family members are represented on the ATH1 microarrays. In short days, 7 out of 11 cycle and 6 of these are phased to 18-19 hours after dawn (Fig. 2B). In thermocycles (LLHC), 8 out of 11 cycle and 4 of these are phased to 8 hours after dawn (not shown in figure). These examples illustrate how diurnal expression profiles can help clarify potential functional redundancies among subgroups within a gene family.

\section{HAYSTACK: A MODEL-BASED PATTERN- MATCHING ALGORITHM}

HAYSTACK (http://haystack.cgrb.oregonstate.edu/) is a program for identifying genes whose expression levels behave similarly across all samples in a microarray data set. Multiple approaches have been developed for organizing and inferring patterns emerging from microarray data (for review, see Belacel et al. 2006). Conventional microarray-clustering approaches are based on identifying distinct or separable groups of genes based on a distance metric (Hierarchical, K-Means, Self-organizing Maps, Support Vector Machines), or principal component analysis. In contrast, our approach is hypothesis-driven and depends on predefined models to identify statistically similar groups of coexpressed genes. By identifying rare occurrences of specific biologically relevant expression patterns in the experimental data, we are able to dramatically reduce the search space for subsequent analyses, including promoter analysis to identify important cis-regulatory elements.

HAYSTACK uses a pattern-matching algorithm to identify genes whose expression patterns fit a user-defined model. HAYSTACK is designed to find rare occurrences of very specific patterns in a large data set and provides an alternative method for clustering microarray data, by grouping genes whose expression patterns match the same or similar HAYSTACK patterns. The algorithm is based on determining the Pearson correlation coefficient between gene expression profiles and user-defined models. HAYSTACK determines the correlation of an experimental data series with each supplied model pattern and applies a series of statistical tests and ad hoc filters to identify genes of interest and their corresponding best-fitting model. We have used HAYSTACK to compare microarray time course data against a collection of diurnal/circadian models to identify cycling genes. The Web version of HAYSTACK (http://haystack.cgrb.oregonstate.edu/) can be used to compare a large-scale data set against a set of user-supplied model patterns to search for biologically relevant patterns in the data.

We are interested in time-of-day specific and circadian transcriptional networks; therefore, we have focused on time course data to highlight the simplicity and power of HAYSTACK. We developed multiple cycling patterns based on diurnal and circadian time courses available in the literature: asymmetric, rigid, spike, cosine, sine, and/or box-like patterns (Harmer et al. 2000; Smith et al. 2004; Blasing et al. 2005; Edwards et al. 2006). To capture both cycling and phase information in the time course data, HAYSTACK patterns were used to mine data from 11 Arabidopsis time courses. The resulting analyses are available through the DIURNAL interface.

To use HAYSTACK, a user starts by uploading a file containing the model patterns (which can be easily constructed using a text editor or Microsoft Excel) and a file containing the microarray data of interest, arranged as a data series in the same format as the model patterns. The user then selects optional statistical criteria and ad hoc filters, and HAYSTACK calculates the correlation coefficient between the expression values across the microarray 

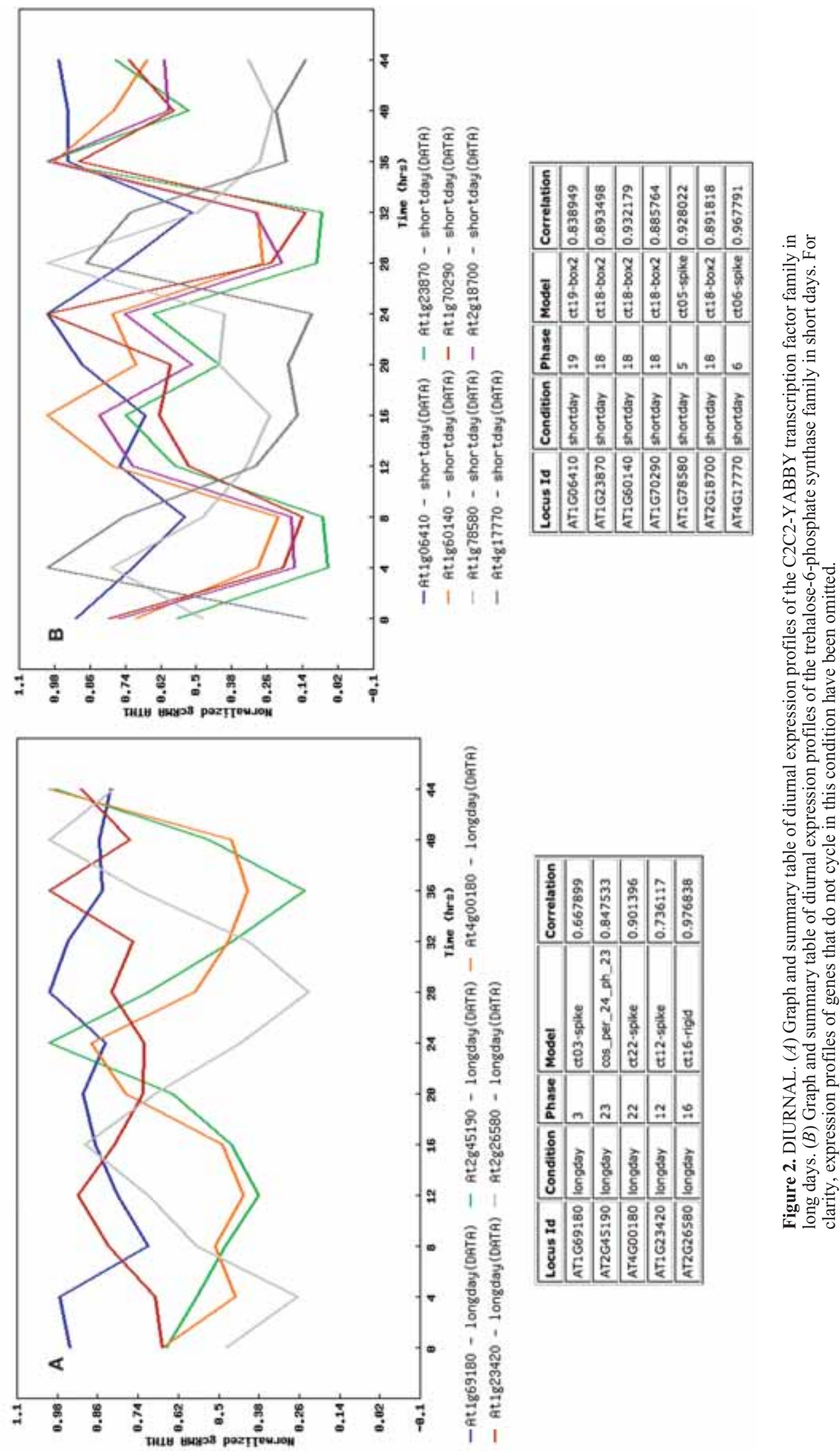
data set for that gene and each of the user-supplied models. HAYSTACK is not limited to any particular microarray platform, genome annotation, or even expression values. It can be used to compare any large-scale data set representing at least three samples (e.g., treatments, genotypes, and time points) against the user-supplied model patterns. A minimum of three data points are necessary for the program to perform valid correlation calculations between the model and experimental data series. The program returns to the user (via e-mail) a link to a results file containing those genes satisfying the user-specified criteria (Fig. 3A). The results from HAYSTACK may be viewed in additional ways. First, the results can be passed directly to a plotting program on the HAYSTACK Web site for easy visual inspection of the data series and corresponding best-match model (Fig. 3B). Alternatively, the results are available in a text file format that may be downloaded and viewed separately.

HAYSTACK can be applied intuitively in several different ways by a biologist. For example, to generate leads for further characterization in the laboratory, a researcher may want to identify all genes in a microarray data set that exhibit an expression profile similar to a particular pattern. Alternatively, a researcher may want to identify genes coexpressed with a particular gene of interest. In this case, the supplied model pattern could be the expression profile of the gene of interest. A third use for HAYSTACK is to seed promoter analysis using ELEMENT or other promoter element discovery programs such as Promomer (Toufighi et al. 2005), SIFT (Hudson and Quail 2003), or MotifSampler (Thijs et al. 2001, 2002). An interesting potential use of HAYSTACK

Haystack Results Page

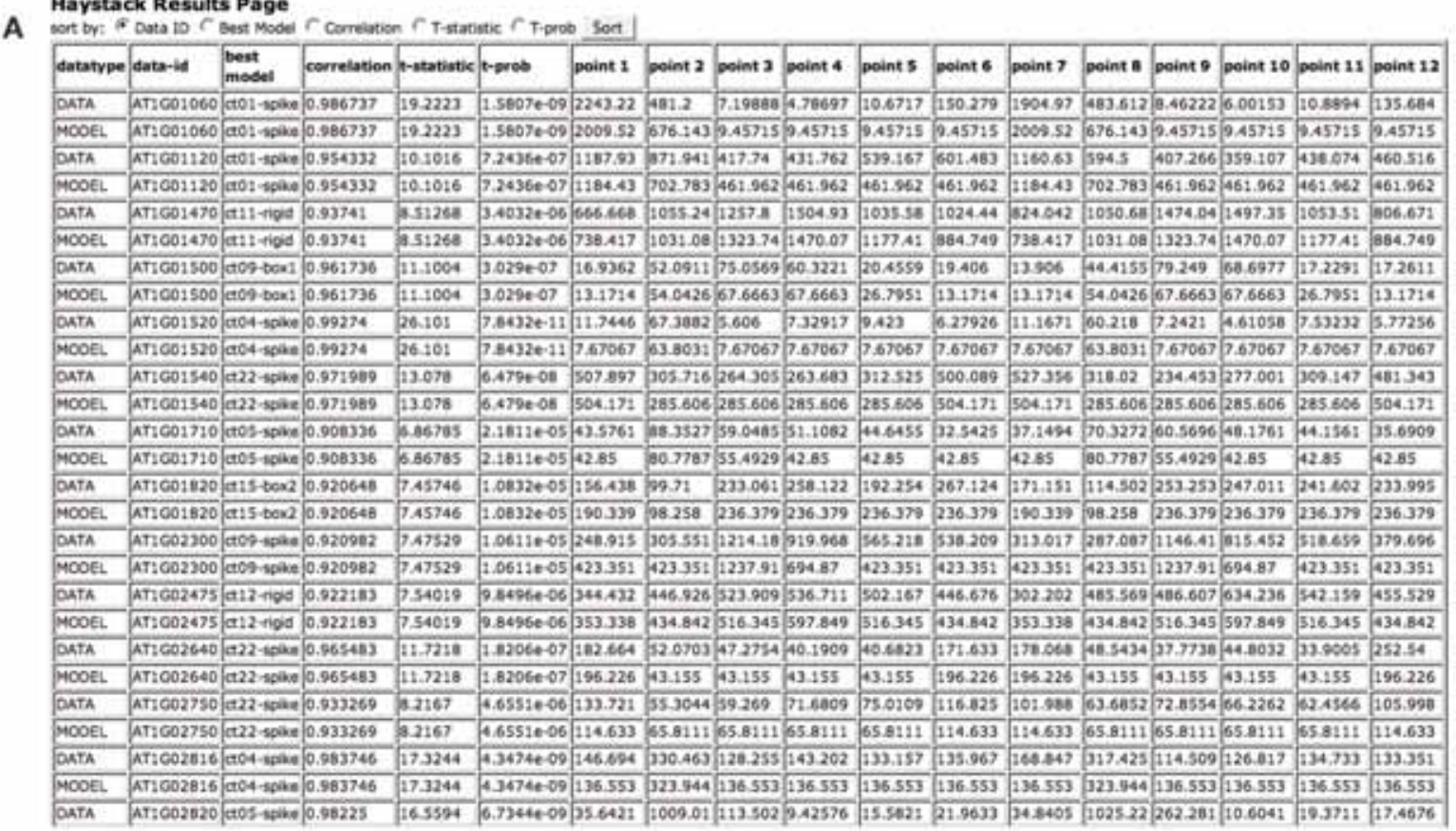

B

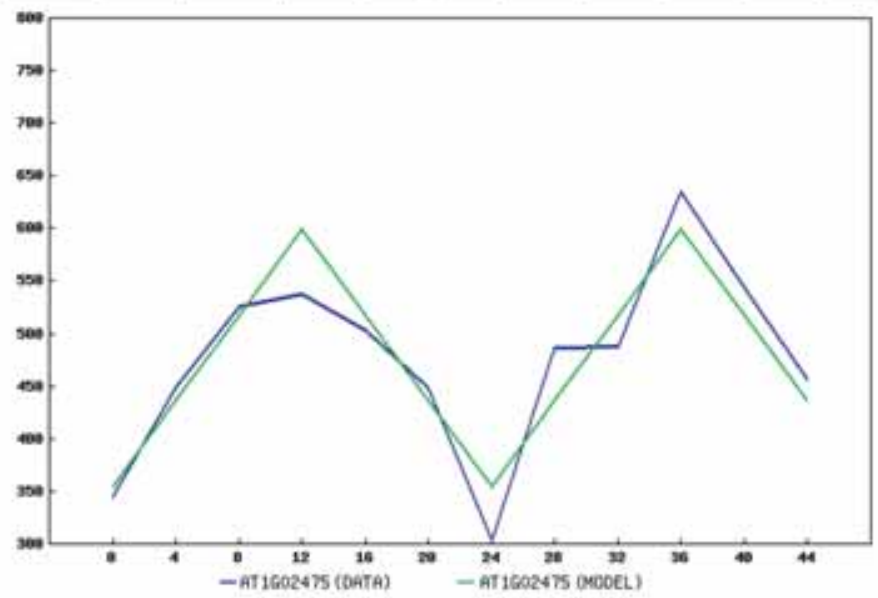

Figure 3. HAYSTACK. (A) Example of an HTML table of HAYSTACK output showing genes, their best matching models, and summary statistics. $(B)$ Example of a graph of gene expression profile and best-fitting HAYSTACK model, derived by clicking on the row of data for a gene shown on the HTML table in $A$. 
involves using it to search nonmicroarray data such as the significance statistics for potential cis elements generated by enumerative promoter-searching tools such as ELEMENT. We used this approach to search the serialized Zscores for overrepresented words identified in our diurnal/circadian studies and thus identified co-occurring elements that form the basis of diurnal/circadian transcriptional network modules (see Fig. 5 below) (T. Michael et al., in prep.).

Other Web-based bioinformatics tools such as the "Expression Angler" or "Sample Angler" at BAR (Toufighi et al. 2005) can identify genes that respond similarly across samples (i.e., genes whose expression profiles are highly correlated), whereas HAYSTACK provides a model-based method for clustering microarray data-genes that are returned with best matches to the same model are potentially coregulated. Some qualifications must be made regarding the results from HAYSTACK, and some of the user options are intended to overcome these shortcomings. For example, spurious model/data matches involving data series in which all of the points are below the background level (noise) are possible. To address this possibility, we implemented a "Background Cutoff," which is the minimum acceptable value for the highest value in the data series. Another possible basis for spurious matches involves situations in which a data series is highly correlated with a model, but the difference between the maximum and minimum values in the data series is insignificant. This problem arises because the Pearson correlation is amplitude-independent. We address this issue by providing a "Fold Cutoff" option that allows a user to determine the minimum acceptable fold difference (i.e., $\max / \mathrm{min}$ ) between the maximum and minimum values in the data series. These options provide flexibility for users to decide what parameters make sense in the context of their experiments.

\section{ELEMENT: A TOOL FOR IDENTIFYING POTENTIAL $C I S$-REGULATORY ELEMENTS IN PLANTS}

The regulation of gene expression in eukaryotes is largely mediated by transcription factors (TFs) that bind within regulatory regions (promoters) upstream of the coding sequence. Transcription factors recognize specific DNA motifs, bind, and in turn interact with each other and the basal transcriptional machinery to regulate the expression of adjacent genes. With the recent availability of high-quality sequenced and annotated genomes, large public microarray databases, and easy access to microarray technology for individual laboratories, there is a need for bioinformatics tools to predict components of transcriptional networks, including transcription-factor-binding sites. Groups of coexpressed genes identified using microarrays may be coregulated and thus can form the foundation for analyses of promoter sequences to identify important cis-regulatory elements.

A number of algorithms have been developed to identify known and putative regulatory elements in the promoter sequences of coregulated genes (for review, see Rombauts et al. 2003; Tompa et al. 2005). The fundamental assump- tion underlying all of these computational approaches is that coregulated genes should contain similar regulatory motifs in their promoters, and these motifs should be significantly overrepresented in the set of coregulated promoters. There are two general computational approaches for identifying potential cis-regulatory elements. One approach is an enumerative method, and the other is an alignment method. The alignment methods are exemplified by programs that use a Gibbs sampling method (Thijs et al. 2002).

The enumerative methods estimate the probability of occurrence of short DNA sequences, or "words," by comparing the count in a set of coregulated sequences to an expected count based on random sampling or statistical modeling of a background distribution (van Helden et al. 1998; Hudson and Quail 2003; Kreps et al. 2003; MarinoRamirez et al. 2004; Nemhauser et al. 2004; Koussevitzky et al. 2007). Therefore, each algorithm requires some background model to calculate an expected frequency for each word. The composition of the sequences underlying the background model is critical because the various features (e.g., exons, introns, and intergenic regions) within a genome exhibit different oligomer compositions. Both enumerative and alignment approaches have been applied to promoter analysis in plants, and the putative coregulated sequences chosen for analysis were typically derived from hierarchical clustering or other analyses of microarray data (Harmer et al. 2000; Chen et al. 2002; Hudson and Quail 2003; Hulzink et al. 2003; Nemhauser et al. 2004; Koussevitzky et al. 2007).

The goal of the ELEMENT program is to provide a user-friendly Web-based tool that uses the enumerative method to identify statistically overrepresented 3-8 mer DNA words in a group of coexpressed genes in Arabidopsis, rice, or poplar. An earlier version of ELEMENT (Nemhauser et al. 2004) calculated Z-scores for each DNA word based on a comparison of the number of occurrences of that word in the upstream sequences of the coexpressed genes against a background distribution derived by random sampling of the upstream sequences of all genes represented on the microarray. The current Webbased version of ELEMENT has been improved considerably. It supports Arabidopsis, poplar, and rice and allows a user to choose various promoter lengths for analysis and to apply a false discovery rate (FDR) filter as desired (Benjamini and Hochberg 1995; Storey and Tibshirani 2003). There are currently a few other Web-based enumerative promoter analysis tools for Arabidopsis, such as the BAR Promomer (Toufighi et al. 2005), SIFT (Hudson and Quail 2003), and TAIR's motiffinder (http://www. arabidopsis.org/tools/bulk/motiffinder/index.jsp). Besides ELEMENT, we are aware of no other Web-based bioinformatics tools for analysis of rice and poplar promoters.

The ELEMENT platform consists of databases of putative Arabidopsis, rice, and poplar regulatory DNAs, word statistics for all 3-8 mer DNA words occurring in these promoter sequences, software implemented in Perl to analyze promoters and apply statistical screening criteria, a series of accessory scripts to summarize the results of these analyses, and a Web interface implemented in Mason and HTML. ELEMENT uses a database of precalculated statistics for 3-8 mer words in the promoters of all genes repre- 
sented on the Arabidopsis, rice, and poplar Affymetrix arrays to estimate the $Z$-score, a measure of the distance in standard deviations of a sample from the mean, for each word. The observed frequency of a particular 3-8 mer word in a group of promoters is compared with the expected frequency of that word derived from the promoters of randomly sampled genes represented on the Affymetrix microarrays for the species, thus providing a background model based on sequences of an appropriate composition for the species and promoter length under consideration. For example, the background model statistics for Oryza sativa ssp. Japonica are derived from the frequencies of all 3-8 mer words in the upstream sequences of 34,967 nontransposable-element-related Japonica rice genes represented on the Affymetrix rice microarrays.

ELEMENT takes as an input a list of standard locus identifiers for the respective species. We have also provided direct links from DIURNAL and ORTHOMAP so that lists of coexpressed genes or groups of predicted orthologs/ homologs may be sent directly to ELEMENT for analysis. The user selects the species and promoter length to be analyzed and adjusts the false-discovery rate and minimum number of occurrences for overrepresented elements as desired. ELEMENT uses, as its reference sets, the $500 \mathrm{bp}$, $1 \mathrm{kbp}, 2 \mathrm{kbp}$, and $3 \mathrm{kbp}$ upstream of annotated gene models of Arabidopsis, rice, and poplar. Because ELEMENT relies on genome annotations, and the transcription start sites are not always well annotated, some gene models lack annotated $5^{\prime}$-untranslated regions (5'UTRs), and thus, in some cases the $3^{\prime}$ end of the predicted promoter region is defined as the beginning of the open reading frame.

ELEMENT returns to the user (via e-mail) a link to results files containing (1) a table of overrepresented elements and their corresponding statistics, with any userselected filtering criteria applied (Fig. 4A), and (2) a

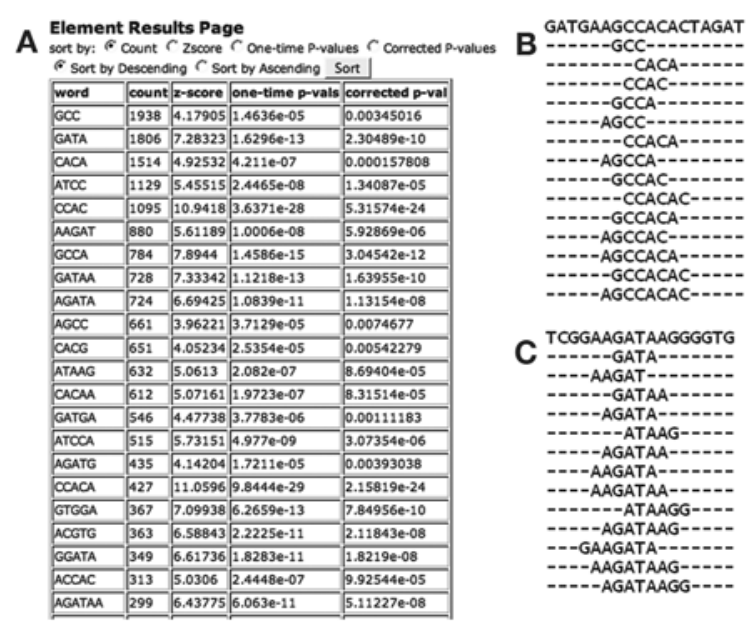

Figure 4. ELEMENT. (A) Example of an HTML table of ELEMENT output showing statistically overrepresented cis elements in a set of 347 promoters of Arabidopsis genes up-regulated in gun1 and gun 5 mutants (Koussevitzky et al. 2007). (B) Close-up of ELEMENT visual output showing alignment of a cluster of CCAC-containing overrepresented words in the promoter of AT2G35960. (C) Close-up of ELEMENT visual output showing alignment of a cluster of GATA-containing overrepresented words in the promoter of AT1G22630. visual alignment of the significant elements aligned to the promoter sequences (Fig. 4B). The latter analysis enables a user to easily identify clusters of nested or partially overlapping overrepresented words or groups of significant words and their relative positions within the promoters. A user may also decide to apply no filtering, in which case, the program returns the statistics for all 3-8 mers.

Results from ELEMENT may be viewed in several ways. First, the results can be viewed on the ELEMENT Web site in an HTML table format (Fig. 4A) that can be sorted online by word, number of occurrences, or significance statistics ( $Z$-score, $p$-value, or corrected $p$-value). Alternatively, the results are available as a text file that may be downloaded for import into a spreadsheet or other program. The results tables also flag overrepresented elements that match known cis elements in the PLACE (Higo et al. 1998) or PlantCARE (Rombauts et al. 1999) databases. Therefore, in addition to predicting novel promoter elements, ELEMENT also finds known cis elements.

To demonstrate the utility of ELEMENT, we have used it to identify both known and novel regulatory motifs that function in the diurnal/circadian regulation of Arabidopsis gene expression. We analyzed 11 diurnal and circadian time courses in the reference plant Arabidopsis. By using HAYSTACK, we were able to identify putative coexpressed/coregulated genes for each phase of the day. The list of genes in each phase served as the input for ELEMENT, which identified overrepresented 3-8 mer DNA words in 500-bp upstream regions.

Our ELEMENT analysis revealed multiple variants of the Morning Element (ME) (Fig. 5A) (Harmer and Kay 2005), Evening Element (EE), and GATA (Fig. 5B) (Schindler and Cashmore 1990; Anderson and Kay 1995; Harmer et al. 2000), and G-box (Fig. 5C) (Giuliano et al. 1988; Michael and McClung 2002, 2003; Hudson and Quail 2003), all promoter elements previously characterized as being involved in light or circadian regulation of gene expression. ELEMENT also predicted previously unknown $c i s$-regulatory elements not identified by other methods (T. Michael et al., in prep.). We have validated a group of these predicted elements using in vivo luciferase imaging, specifying their activity to a phase of day during which no diurnal- or circadian-associated cis elements had previously been identified. These predictions of novel diurnal/circadian-associated elements were robust enough to be well-conserved in rice and poplar. Therefore, using ELEMENT, we were able to predict both known and novel cis-regulatory elements and define specific aspects of their activity that were previously unknown.

\section{CONCLUSIONS AND FUTURE DIRECTIONS}

In conclusion, DIURNAL, HAYSTACK, and ELEMENT are a multifunctional, user-friendly, and complementary collection of Web-based tools. Our Affymetrix microarray data for plant diurnal and circadian time courses are accessible through the DIURNAL interface, which provides a powerful means to query and visualize the data. Two additional tools, HAYSTACK and ELEMENT, provide useful methods for querying microarray data. HAYSTACK analysis constitutes an alternative 


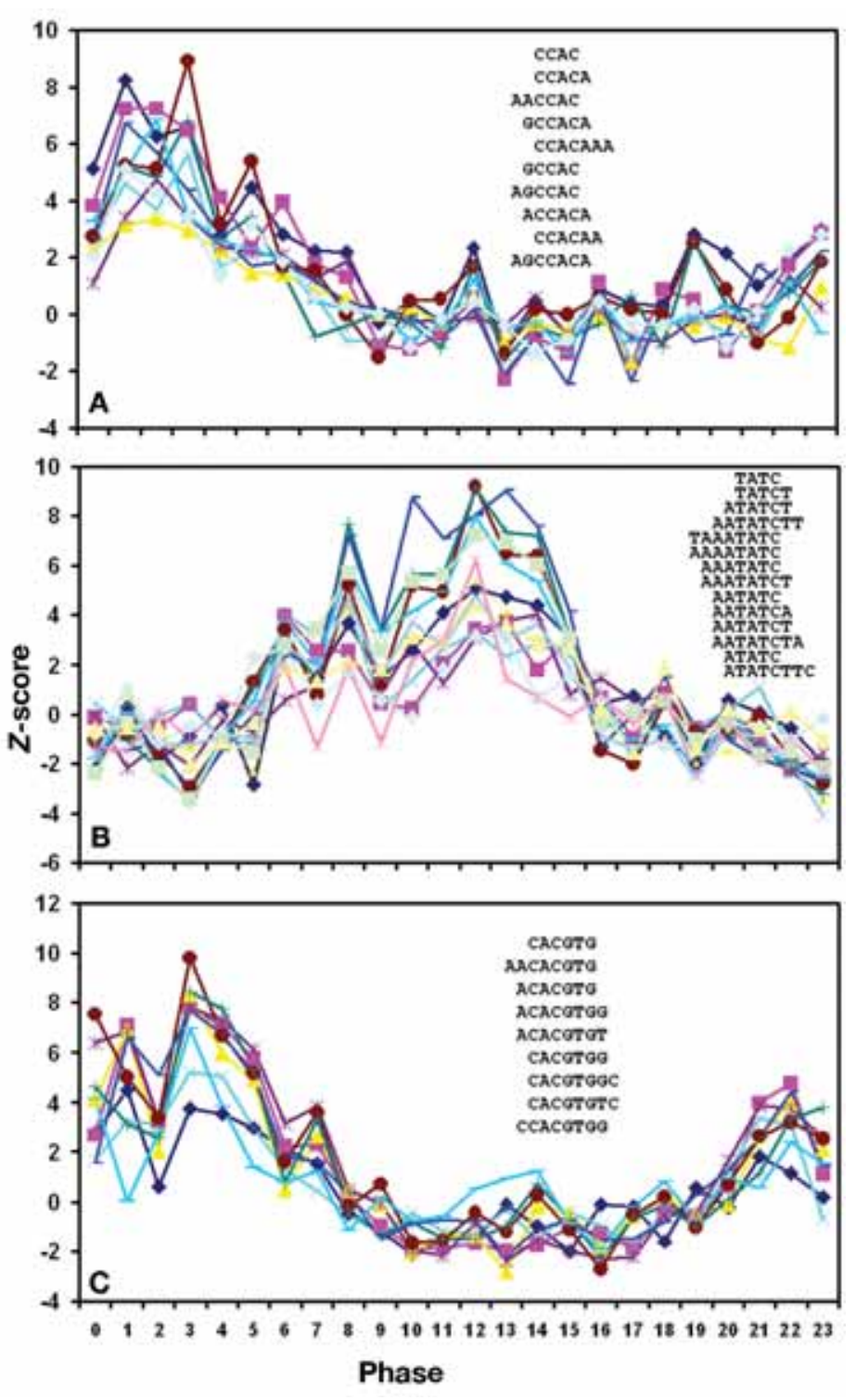

Figure 5. Examples of $Z$-score profiles and overrepresented word clusters for known light- and circadian-associated motifs under diurnal conditions. (A) Cluster of overrepresented words and their $Z$ score profiles under the light/dark (12:12) condition (LDHH_SM). The clustered words correspond to Morning Element (ME) variants. $(B)$ A cluster of overrepresented words and their $Z$-score profiles under the long-day condition. The clustered words represent Evening Element (EE) and GATA variants. $(C)$ A cluster of overrepresented words and their Z-score profiles under the short-day condition. The clustered words represent G-Box variants. method for clustering microarray data by grouping together genes whose expression patterns match the same or similar user-defined HAYSTACK patterns. HAYSTACK can be used to compare virtually any largescale data set against a set of user-supplied model patterns. ELEMENT identifies predicted cis-regulatory elements within the promoters of groups of coexpressed genes from Arabidopsis, rice, or poplar and can be used to facilitate cross-species comparisons of promoter architecture.

Data from our poplar and rice microarray time courses will be added to the DIURNAL database over the next year. We plan to add diurnal and circadian time course data for additional species when such data become available. We also plan to expand ELEMENT to support promoter analysis for additional plant species with sequenced genomes or genomes that will be sequenced in the near future, including sorghum, medicago, maize, Arabidopsis lyrata, papaya, and Brachypodium distachyon. We hope that these resources will help to accelerate functional genomics efforts directed at the mechanisms underlying diurnal and circadian biology in plants. For example, in Arabidopsis, the ability to order knockout or knockdown T-DNA insertion lines directly from stock centers makes it possible to rapidly perform in vivo tests of in silico predictions derived from HAYSTACK analysis of microarray data. Promoter element predictions from ELEMENT can in turn be tested experimentally to identify functional cis-regulatory elements.

\section{ACKOWLEDGMENTS}

This work was supported by a National Science Foundation Plant Genome grant (DBI 0605240) (T.C.M, J.C., and S.A.K), the Howard Hughes Medical Institute, and National Institutes of Health (NIH) grants GM56006 and GM67837 (S.A.K.) and GM52413 and GM62932 (J.C.). T.P.M and T.C.M were supported by Ruth L. Kirschstein NIH Postdoctoral Fellowships. 


\section{REFERENCES}

Anderson S.L. and Kay S.A. 1995. Functional dissection of circadian clock- and phytochrome-regulated transcription of the Arabidopsis CAB2 gene. Proc. Natl. Acad. Sci. 92: 1500.

Arabidopsis Genome Initiative. 2000. Analysis of the genome sequence of the flowering plant Arabidopsis thaliana. Nature 408: 796.

Barrett T., Troup D.B., Wilhite S.E., Ledoux P., Rudnev D., Evangelista C., Kim I.F., Soboleva A., Tomashevsky M., and Edgar R. 2007. NCBI GEO: Mining tens of millions of expression profiles: Database and tools update. Nucleic Acids Res. (database issue) 35: D760.

Belacel N., Wang Q., and Cuperlovic-Culf M. 2006. Clustering methods for microarray gene expression data. OMICS 10: 507.

Benjamini Y. and Hochberg Y. 1995. Controlling the false discovery rate: A practical and powerful approach to multiple testing. J. R. Stat. Soc. B 57: 289.

Blasing O., Gibon Y., Gunther M., Hohne M., Morcuende R., Osuna D., Thimm O., Usadel B., Scheible W., and Stitt M. 2005. Sugars and circadian regulation make major contributions to the global regulation of diurnal gene expression in Arabidopsis. Plant Cell 17: 3257.

Brazma A., Parkinson H., Sarkans U., Shojatalab M., Vilo J., Abeygunawardena N., Holloway E., Kapushesky M., Kemmeren P., Lara G.G., Oezcimen A., Rocca-Serra P., and Sansone S.A. 2003. ArrayExpress: A public repository for microarray gene expression data at the EBI. Nucleic Acids Res. 31: 68 .

Chen W., Provart N.J., Glazebrook J., Katagiri F., Chang H.S., Eulgem T., Mauch F., Luan S., Zou G., Whitham S.A., Budworth P.R., Tao Y., Xie Z., Chen X., Lam S., Kreps J.A., Harper J.F., Si-Ammour A., Mauch-Mani B., Heinlein M., Kobayashi K., Hohn T., Dangl J.L., Wang X., and Zhu T. 2002. Expression profile matrix of Arabidopsis transcription factor genes suggests their putative functions in response to environmental stresses. Plant Cell 14: 559.

Craigon D.J., James N., Okyere J., Higgins J., Jotham J., and May S. 2004. NASCArrays: A repository for microarray data generated by NASC's transcriptomics service. Nucleic Acids Res. (database issue) 32: D575.

Dodd A.N., Salathia N., Hall A., Kevei E., Toth R., Nagy F., Hibberd J.M., Millar A.J., and Webb A.A. 2005. Plant circadian clocks increase photosynthesis, growth, survival, and competitive advantage. Science 309: 630.

Edwards K.D., Anderson P.E., Hall A., Salathia N.S., Locke J.C., Lynn J.R., Straume M., Smith J.Q., and Millar A.J. 2006. FLOWERING LOCUS C mediates natural variation in the high-temperature response of the Arabidopsis circadian clock. Plant Cell 18: 639.

Fraser H.B., Hirsh A.E., Wall D.P., and Eisen M.B. 2004. Coevolution of gene expression among interacting proteins. Proc. Natl. Acad. Sci. 101: 9033.

Garcia-Hernandez M., Berardini T.Z., Chen G., Crist D., Doyle A., Huala E., Knee E., Lambrecht M., Miller N., Mueller L.A., Mundodi S., Reiser L., Rhee S.Y., Scholl R., Tacklind J., Weems D.C., Wu Y., Xu I., Yoo D., Yoon J., and Zhang P. 2002. TAIR: A resource for integrated Arabidopsis data. Funct. Integr. Genomics 2: 239.

Giuliano G., Pichersky E., Malik V.S., Timko M.P., Scolnik P.A., and Cashmore A.R. 1988. An evolutionarily conserved protein binding sequence upstream of a plant light-regulated gene. Proc. Natl. Acad. Sci. 85: 7089

Harmer S. and Kay S. 2005. Positive and negative factors confer phase-specific circadian regulation of transcription in Arabidopsis. Plant Cell 17: 1926.

Harmer S.L., Hogenesch J.B., Straume M., Chang H.-S., Han B., Zhu T., Wang X., Kreps J.A., and Kay S.A. 2000. Orchestrated transcription of key pathways in Arabidopsis by the circadian clock. Science 290: 2110.

Higo K., Ugawa Y., Iwamoto M., and Higo H. 1998. PLACE: A database of plant cis-acting regulatory DNA elements. Nucleic Acids Res. 26: 358.
Hudson M. and Quail P. 2003. Identification of promoter motifs involved in the network of phytochrome A-regulated gene expression by combined analysis of genomic sequence and microarray data. Plant Physiol. 133: 1605.

Hulzink R.J., Weerdesteyn H., Croes A.F., Gerats T., van Herpen M.M., and van Helden J. 2003. In silico identification of putative regulatory sequence elements in the 5 '-untranslated region of genes that are expressed during male gametogenesis. Plant Physiol. 132: 75.

International Rice Genome Sequencing Project. 2005. The mapbased sequence of the rice genome. Nature 436: 793.

Karim S., Aronsson H., Ericson H., Pirhonen M., Leyman B., Welin B., Mantyla E., Palva E.T., Van Dijck P., and Holmstrom K.O. 2007. Improved drought tolerance without undesired side effects in transgenic plants producing trehalose. Plant Mol. Biol. 64: 371 .

Koussevitzky S., Nott A., Mockler T.C., Hong F., SachettoMartins G., Surpin M., Lim J., Mittler R., and Chory J. 2007. Signals from chloroplasts converge to regulate nuclear gene expression. Science 316: 715.

Kreps J., Budworth P., Goff S., and Wang R. 2003. Identification of putative plant cold responsive regulatory elements by gene expression profiling and a pattern enumeration algorithm. Plant Biotechnol. J. 1: 345.

Leyman B., Van Dijck P., and Thevelein J.M. 2001. An unexpected plethora of trehalose biosynthesis genes in Arabidopsis thaliana. Trends Plant Sci. 6: 510.

Marino-Ramirez L., Spouge J.L., Kanga G.C., and Landsman D. 2004. Statistical analysis of over-represented words in human promoter sequences. Nucleic Acids Res. 32: 949.

Michael T.P. and McClung C.R. 2002. Phase-specific circadian clock regulatory elements in Arabidopsis. Plant Physiol. 130: 627.

2003. Enhancer trapping reveals widespread circadian clock transcriptional control in Arabidopsis thaliana. Plant Physiol. 132: 629 .

Michael T.P., Salome P.A., Yu H.J., Spencer T.R., Sharp E.L., McPeek M.A., Alonso J.M., Ecker J.R., and McClung C.R. 2003. Enhanced fitness fonferred by naturally occurring variation in the circadian clock. Science 302: 1049.

Nemhauser J.L., Mockler T.C., and Chory J. 2004. Interdependency of brassinosteroid and auxin signaling in Arabidopsis. PLoS Biol. 2: e258.

Rhee S.Y., Beavis W., Berardini T.Z., Chen G., Dixon D., Doyle A., Garcia-Hernandez M., Huala E., Lander G., Montoya M., Miller N., Mueller L.A., Mundodi S., Reiser L., Tacklind J., Weems D.C., Wu Y., Xu I., Yoo D., Yoon J., and Zhang P. 2003. The Arabidopsis Information Resource (TAIR): A model organism database providing a centralized, curated gateway to Arabidopsis biology, research materials and community. Nucleic Acids Res. 31: 224.

Rombauts S., Dehais P., Van Montagu M., and Rouzé P. 1999. PlantCARE, a plant cis-acting regulatory element database. Nucleic Acids Res. 27: 295.

Rombauts S., Florquin K., Lescot M., Marchal K., Rouzé P., and van de Peer Y. 2003. Computational approaches to identify promoters and cis-regulatory elements in plant genomes. Plant Physiol. 132: 1162.

Schindler U. and Cashmore A.R. 1990. Photoregulated gene expression may involve ubiquitous DNA binding proteins. EMBO J. 9: 3415 .

Schmid M., Davison T., Henz S., Pape U., Demar M., Vingron M., Scholkopf B., Weigel D., and Lohmann J. 2005. A gene expression map of Arabidopsis thaliana development. Nat. Genet. 37: 501.

Shen L., Gong J., Caldo R.A., Nettleton D., Cook D., Wise R.P., and Dickerson J.A. 2005. BarleyBase: An expression profiling database for plant genomics. Nucleic Acids Res. (database issue) 33: D614.

Siegfried K.R., Eshed Y., Baum S.F., Otsuga D., Drews G.N., and Bowman J.L. 1999. Members of the YABBY gene family specify abaxial cell fate in Arabidopsis. Development 126: 4117.

Smith S., Fulton D., Chia T., Thorneycroft D., Chapple A., Dunstan H., Hylton C., Zeeman S., and Smith A. 2004. 
Diurnal changes in the transcriptome encoding enzymes of starch metabolism provide evidence for both transcriptional and posttranscriptional regulation of starch metabolism in Arabidopsis leaves. Plant Physiol. 136: 2687.

Storey J.D. and Tibshirani R. 2003. Statistical significance for genomewide studies. Proc. Natl. Acad. Sci. 100: 9440.

Thijs G., Lescot M., Marchal K., Rombauts S., De Moor B., Rouzé P., and Moreau Y. 2001. A higher-order background model improves the detection of promoter regulatory elements by Gibbs sampling. Bioinformatics 17: 1113.

Thijs G., Marchal K., Lescot M., Rombauts S., De Moor B., Rouzé P., and Moreau Y. 2002. A Gibbs sampling method to detect overrepresented motifs in the upstream regions of coexpressed genes. J. Comput. Biol. 9: 447.

Tompa M., Li N., Bailey T.L., Church G.M., De Moor B., Eskin E., Favorov A.V., Frith M.C., Fu Y., Kent W.J., Makeev V.J., Mironov A.A., Noble W.S., Pavesi G., Pesole G., Regnier M., Simonis N., Sinha S., Thijs G., van Helden J., Vandenbogaert M., Weng Z., Workman C., Ye C., and Zhu Z. 2005. Assessing computational tools for the discovery of transcription factor binding sites. Nat. Biotechnol. 23: 137.
Toufighi K., Brady S.M., Austin R., Ly E., and Provart N.J. 2005. The Botany Array Resource: e-Northerns, Expression Angling, and promoter analyses. Plant J. 43: 153.

Tuskan G.A., DiFazio S., Jansson S., Bohlmann J., Grigoriev I., Hellsten U., Putnam N., Ralph S., Rombauts S., Salamov A., Schein J., Sterck L., Aerts A., Bhalerao R.R., Bhalerao R.P., Blaudez D., Boerjan W., Brun A., Brunner A., Busov V., Campbell M., Carlson J., Chalot M., Chapman J., and Chen G.L., et al. 2006. The genome of black cottonwood, Populus trichocarpa (Torr. \& Gray). Science 313: 1596.

van Helden J., Andre B., and Collado-Vides J. 1998. Extracting regulatory sites from the upstream region of yeast genes by computational analysis of oligonucleotide frequencies. $J$. Mol. Biol. 281: 827.

Woelfle M., Ouyang Y., Phanvijhitsiri K., and Johnson C. 2004. The adaptive value of circadian clocks: An experimental assessment in cyanobacteria. Curr. Biol. 14: 1481.

Zimmermann P., Hirsch-Hoffmann M., Hennig L., and Gruissem W. 2004. GENEVESTIGATOR. Arabidopsis microarray database and analysis toolbox. Plant Physiol. 136: 2621. 


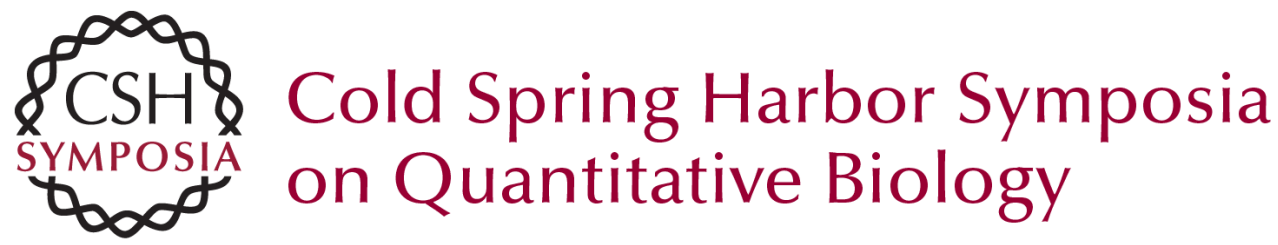

\section{The Diurnal Project: Diurnal and Circadian Expression Profiling, Model-based Pattern Matching, and Promoter Analysis}

T. C. Mockler, T. P. Michael, H. D. Priest, et al.

Cold Spring Harb Symp Quant Biol 2007 72: 353-363

Access the most recent version at doi:10.1101/sqb.2007.72.006

References This article cites 46 articles, 21 of which can be accessed free at: http://symposium.cshlp.org/content/72/353.full.html\#ref-list-1

License

Email Alerting

Receive free email alerts when new articles cite this article - sign up in the box at the Service top right corner of the article or click here.

To subscribe to Cold Spring Harbor Symposia on Quantitative Biology go to: http://symposium.cshlp.org/subscriptions 